\title{
String tension scaling in models of the confined phase
}

Peter N. Meisinger and Michael C. Ogilvie*

Washington University Dept. of Physics, St. Louis, MO 63130 USA

E-mail: pnm@wuphys . wust l . edu, mco@wuphys . wust l . edu

\begin{abstract}
We introduce a D-dimensional Hamiltonian formalism for the study of Polyakov loop models of finite temperature gauge theories in D+1 dimensions. Polyakov loop string tensions are obtained from energy eigenstates of the Hamiltonian. For $\mathrm{D}=1$, the gauge theory reduces to quantum mechanics on the gauge group; for $\mathrm{D}>1$, the Hamiltonian includes hopping terms that link sites on the transverse lattice. The deconfined phase is associated with a ground state which breaks $\mathrm{Z}(\mathrm{N})$ symmetry, and Svetitsky-Yaffe critical universality emerges naturally for $\mathrm{D}>1$. A minimal model is proposed which naturally reproduces approximate Casimir scaling for a range of couplings. Different classes of potentials lead to different pictures of how confinement is realized. Such potential energy terms also modify string tension scaling laws, as we demonstrate using two potentials: one representing the perturbative thermal contributions from gluons, and the other arising from magnetic monopoles in certain confining supersymmetric theories.
\end{abstract}

XXIVth International Symposium on Lattice Field Theory

July 23-28, 2006

Tucson, Arizona, USA

\footnotetext{
*Speaker.
} 


\section{Introduction}

There is a substantial body of evidence from lattice simulations $[1,2,3,4,5,6,7]$ that string tensions in confining gauge theories obey scaling laws. One strong possibility is Casimir scaling, where the ratio of string tensions obey $\sigma_{R^{\prime}} / \sigma_{R}=C_{2 R^{\prime}} / C_{2 R}$ with $C_{2 R}$ the quadratic Casimir for the representation $R$. Another possible scaling law, suggested by string theory, is sine-law scaling, in which the lowest string tension in each $N$-ality sector obeys $\sigma_{k} / \sigma_{1}=\sin (k \pi / N) / \sin (\pi / N)$ $[8,9]$. We will explore string tension scaling using a class of Hamiltonian effective models for Polyakov loops obtained from underlying $D+1$-dimensional gauge theories using the same type of arguments which lead to the Euclidean effective action for Polyakov loops [10]. These models are defined on a $D$-dimensional space with one continuous dimension and $D-1$ discrete directions. The general form of the Hamiltonian may be written as

$$
H=\sum_{j}\left[\kappa C_{2 j}+V_{j}\right]-\frac{J_{F}}{2} \sum_{\langle j k\rangle}\left[\chi_{F j} \chi_{F k}^{*}+\chi_{F j}^{*} \chi_{F k}\right]
$$

The first term is the kinetic term for each site $j$, given by the Casimir operator. Higher-order terms may also be present. The second term is the potential on each site, $V_{j}$, a $Z(N)$-invariant class function of the group. The third term is a hopping term between nearest-neighbor sites, and generalizes to include other representations and longer hops. $H$ acts on wave functionals which are class functions $\Psi[P]$ of Polyakov loops. The Hilbert space is spanned by products of characters, with Haar measure providing the natual inner product. The integration over each Polyakov loop need only be taken over the maximal Abelian subalgebra, so that each Polyakov loop $P_{j}$ may be represented as $P_{j}=\operatorname{diag}\left[e^{i \theta_{1}} . . e^{i \theta_{N}}\right]$. The ground state energy density $E_{0}$ in the Hamiltonian formalism is related to the free energy density $f$ and the pressure $p$ via $\beta f=-\beta p=$ $E_{0}$.

A central question we address is naturalness: what class of potentials lead to Casimir scaling or similar behavior? Many potentials have regions of parameter space which tend to localize wave functions around $N$ degenerate minima related by $Z(N)$ symmetry. In such cases, $Z(N)$ symmetry can be maintained in the confined phase by tunneling. If we have an approximate wave function $\Phi_{0}(P)$ which is localized near one of the degenerate minima, we can construct a set of $N$ wave functions as

$$
\Psi_{k}(P)=\frac{1}{\sqrt{N}} \sum_{j=0}^{N-1}\left[e^{2 \pi i j k / N} \Phi_{0}\left(e^{-2 \pi i j k / N} P\right)\right]
$$

It is easy to show that the string tension splitting in this case is determined by tunneling, giving a scaling law of the form $\sigma_{k} / \sigma_{1}=\sin ^{2}(k \pi / N) / \sin ^{2}(\pi / N)$. Another, complementary limit localizes the wave function around the unique set of eigenvalues left invariant by $\mathrm{Z}(\mathrm{N})$ symmetry. For $S U(3)$, this gives the matrix $P_{0}=\operatorname{diag}\left[1, e^{2 \pi i / 3}, e^{4 \pi i / 3}\right]$. For any representation $R$ with non-zero $N$-ality, $\chi_{R}\left(P_{0}\right)=0$, so a configuration of Polyakov loops of the form $g(x) P_{0} g^{-1}(x)$ can be said to confine. These limiting behaviors represent the extremes of the potentials we discuss below.

\section{Mean Field Theory}

In a Hamiltonian formalism, mean field theory is equivalent to the Hartree approximation. We assume that the ground wave function is a product of uncorrelated site wave functions: $\Psi[P]=$ 


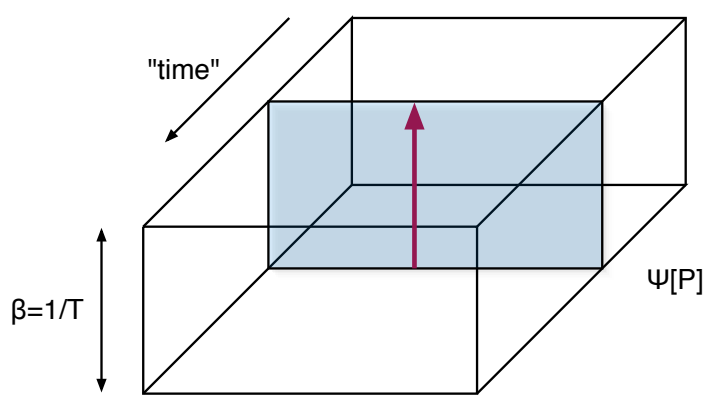

Figure 1: Lattice geometry for Hamiltonian formalism.

$\prod \psi_{j}\left(P_{j}\right)$. Minimizing the energy and requiring self-consistency gives the Hamiltonian $H_{j}$ for the site $j$ :

$$
H_{j}=\kappa C_{2 j}+V_{j}-\frac{J_{F} d}{2}\left[\chi_{F j}\left\langle\chi_{F}^{*}\right\rangle+\chi_{F j}^{*}\left\langle\chi_{F}\right\rangle\right]
$$

In the confined phase, $Z(N)$ symmetry requires $\left\langle\chi_{F}\right\rangle=0$, and we are left with a Hamiltonian for $Z(N)$-invariant quantum mechniacs on the group manifold:

$$
\left[\kappa C_{2 j}+V_{j}\right] \psi_{j n}\left(P_{j}\right)=E_{n} \psi_{j n}\left(P_{j}\right)
$$

As a first approximation, we associate the eigenvalues with string tensions via $E_{n}-E_{0}=\sigma_{n} / T$.

The Hamiltonian formalism is capable of reproducing the behavior of the deconfinement phase transition revealed by familiar models based on an effective action [10]. In the case of $S U(2)$, assume for simplicity a potential $V_{j}=-h_{A} \chi_{A j}$ and a trial wave function of the form $\psi_{0 j}=\sqrt{1-A^{2}-B^{2}}+A \chi_{A j}+B \chi_{F j}$ where $A$ and $B$ can be taken to be real. The order parameter for the deconfinement transition is the expected value of the Polyakov loop in the fundamental representation $\left\langle\chi_{F}\right\rangle \equiv m(A, B)=2 B\left(A+\sqrt{1-A^{2}-B^{2}}\right)$. The ground state energy density is given by the minimum of

$$
E_{v a r}=\kappa\left(C_{A} A^{2}+C_{F} B^{2}\right)-(D-1) J_{F} m^{2}(A, B)-h_{A}\left(A^{2}+2 A+B^{2}\right)
$$

The low-temperature, confined phase occurs when the term in $E_{\text {var }}$ proportional to $\kappa$ dominates, and $E_{v a r}$ is minimized when $B=0$. If $h_{A}$ is non-zero, $A$ will be non-zero, and $\left\langle\chi_{A}\right\rangle$ will have a non-zero expectation value at all temperatures. As the temperature grows, the hopping parameter $J_{F}$ grows as well. When the coefficient of $B^{2}$ in $E_{v a r}$ becomes negative, there is a second-order phase transition to the deconfined phase where $B \neq 0$ and $Z(2)$ symmetry is spontaneously broken. Note that the $h_{A}$ potential term causes the critical temperature to change, but not the order of the transition.

In the case of $S U(3)$, we take a trial wave function of the form $\psi_{0 j}=\sqrt{1-A^{2}-2 B^{*} B}+A \chi_{A j}+$ $B^{*} \chi_{F j}+B \chi_{F j}^{*}$ and the order parameter is given by $\left\langle\chi_{F j}\right\rangle \equiv m(A, B)=2 B\left(A+\sqrt{1-A^{2}-2 B^{*} B}\right)+$ $\left(B^{*}\right)^{2}$. The variational estimate of the ground state energy density is given by

$$
E_{\text {var }}=\kappa\left(C_{A} A^{2}+C_{F} B^{2}\right)-(D-1) J_{F}|m(A, B)|^{2}-h_{A}\left(2 A^{2}+2 A+2 B^{*} B\right)
$$

The new feature in $S U(3)$ is the occurrence of a term proportional to $J_{F}\left(B^{3}+B^{* 3}\right)$. Such a term is permitted by $Z(3)$ symmetry and makes the deconfining transition first order. 


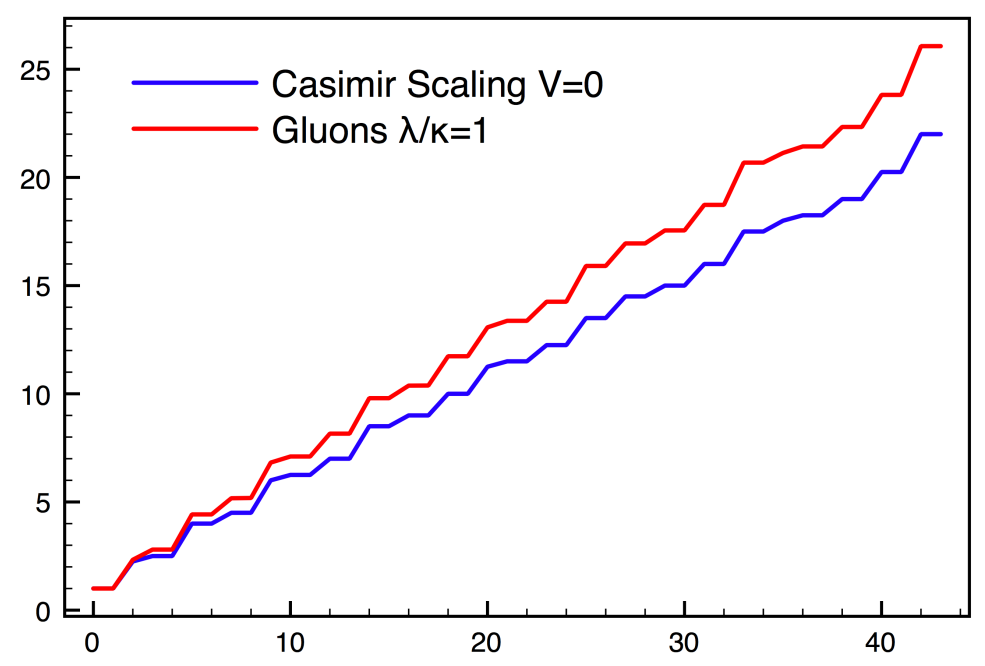

Figure 2: String tension ratios for thermal gluon potential.

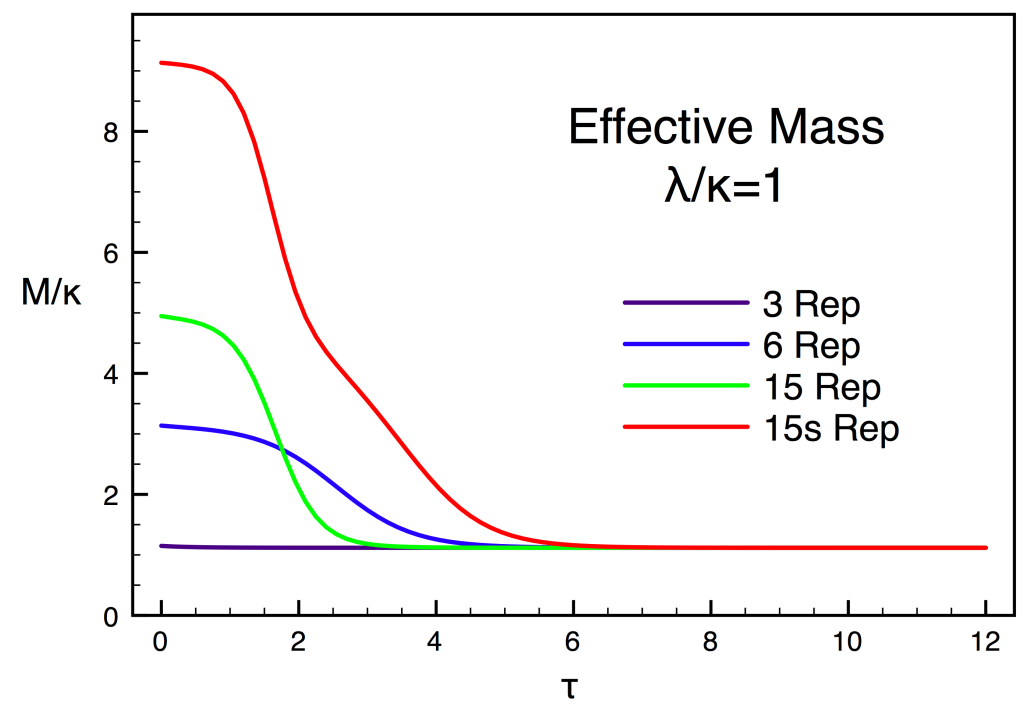

Figure 3: Effective Masses for thermal gluon potential.

\section{Potentials}

Particle loop effects contribute to the potential terms in the effective Hamiltonian. The oneloop contribution of the massless gauge bosons to $V$ is

$$
-\lambda \sum_{n=1}^{\infty} \frac{1}{n^{4}} \chi_{A}\left(P^{n}\right)
$$

with $\lambda=2 T^{4} / \pi^{2}$. This potential is minimized at the elements of $Z(N)$, and yields the familiar black-body formula for the free energy density at those points. We have calculated the string 


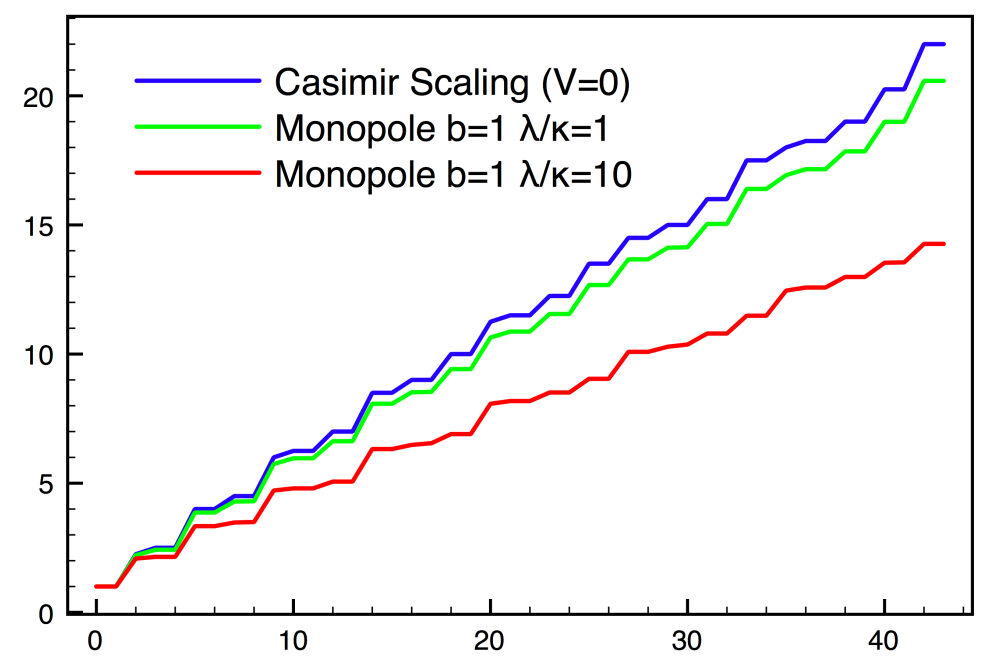

Figure 4: String tension ratios for $W$ at $B=1$.

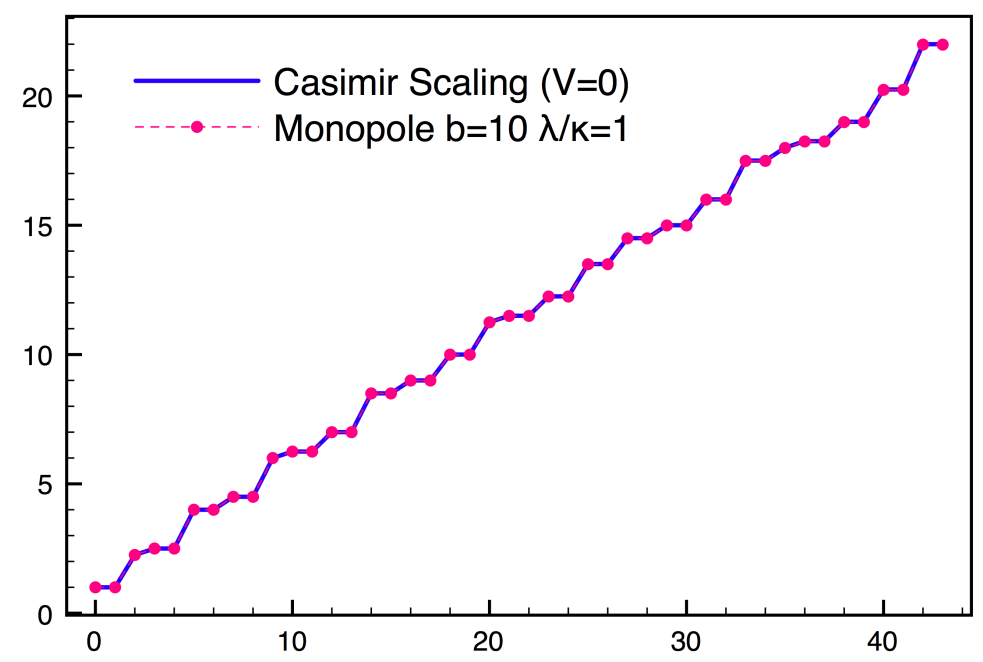

Figure 5: String tension ratios for $W$ at $B=10$.

tensions for this potential by diagonalizing the quantum-mechanical $S U(3)$ Hamiltonian on a finite basis, using as a basis all representations up to eight columns wide in the Young tableaux. In figure 2 we compare the sorted string tension ratios $\sigma_{R} / \sigma_{F}$ for $\lambda / \kappa=1$ with Casimir scaling. Although there are systematic deviations, they are most apparent for larger representations. We can define an effective mass associated with the exponential decay of each representation as a function of the scaled time $\tau=\left(E_{1}-E_{0}\right) t$. Figure 3 shows substantial mixing between representations, as evidenced by the rapid approach of effective masses to the lowest mass.

Topological excitations also contribute to the potential. For example, the one-loop calculation in $S U$ (2) of caloron contributions to the functional integral in $S U(2)$ at finite temperature indicates 


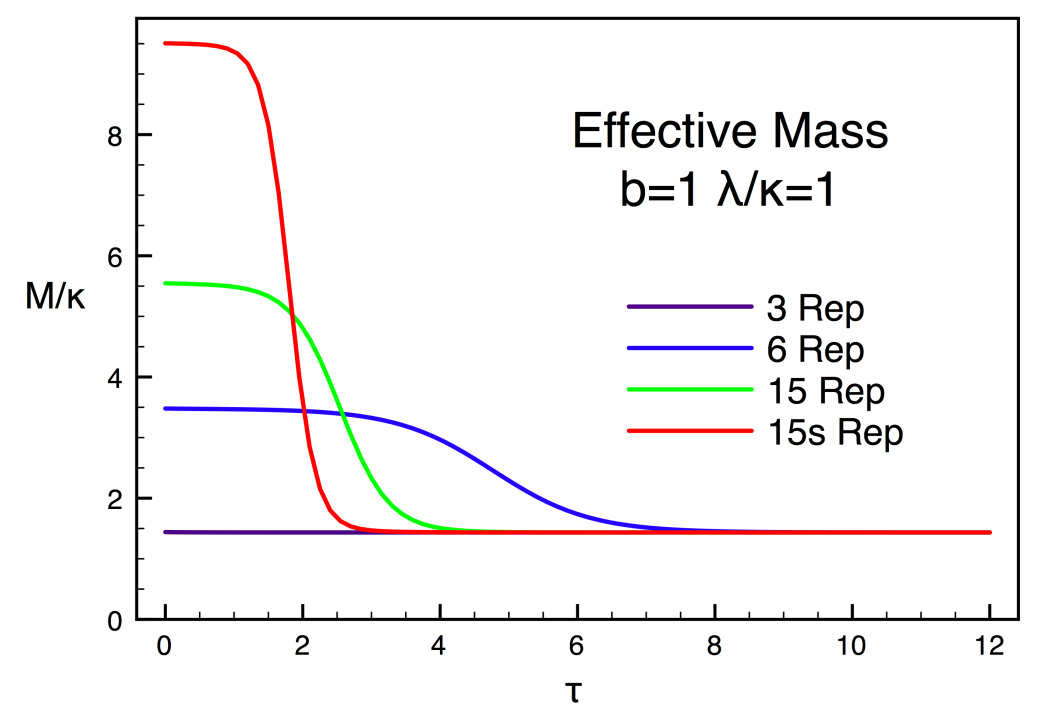

Figure 6: Effective Mass Ratios at $b=1$.

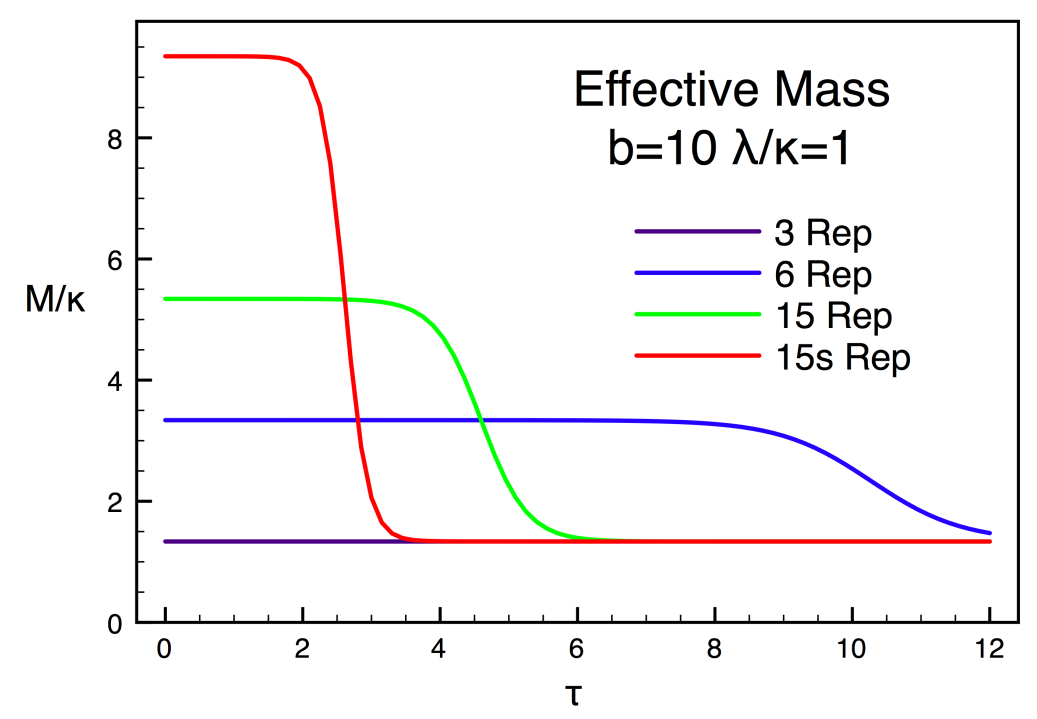

Figure 7: Effective Mass Ratios at $b=10$

an instability of the deconfined plasma phase at sufficiently low temperature [11]. In certain supersymmetric $S U(N)$ gauge theories on $S^{1} \times R^{3}$, confinement can be understoood as arising from magnetic monopole effects [11, 12]. The superpotential $W$, regarded as a function of the Polyakov loop eigenvalues, is the affine Toda potential, and is minimized at $P_{0}$. In the case of $S U(3)$, the superpotential can be written in the form

$$
W=\lambda\left[e^{-b\left(\theta_{1}-\theta_{2}\right)}+e^{-b\left(\theta_{2}-\theta_{3}\right)}+e^{-b\left(2 \pi-\theta_{1}+\theta_{3}\right)}\right]
$$

where $b=4 \pi / g^{2}$. The eigenvalues are taken to lie in the first Weyl chamber, where the eigenvalues are ordered as $\theta_{1} \geq \theta_{2} \geq \theta_{3}$. We will use $W$ as an example of a potential that is minimized at $P_{0}$. 
Figure 4 shows that the deviation from Casimir scaling increases with the strength of the potential $\lambda$ as expected. In comparison, figure 5 indicates that at fixed $\lambda$, Casimir scaling is recovered in the limit of large $b$. As $b$ increases, $V$ is effectively zero except near the boundary between Weyl chambers, where two or more Polyakov loop eigenvalues coincide. Because Haar measure leads to eigenvalue repulsion, the wave function naturally vanishes when two eigenvalues coincide, and for large $b, W$ has little effect on the wave functions.

The effective masses, shown in figures 6 and 7, show longer plateaus at larger $b$. This is particularly noticeable for smaller representations. This is consistent with the wave functions for large $b$ approaching the $V=0$ wave functions. Thus increasing $b$ decreases mixing between representations of the same $N$-ality.

\section{Acknowledgments}

MCO gratefully acknowledges the support of the U.S. Dept. of Energy.

\section{References}

[1] S. Deldar, Phys. Rev. D 62, 034509 (2000) [arXiv:hep-lat/9911008].

[2] G. S. Bali, Phys. Rev. D 62, 114503 (2000) [arXiv:hep-lat/0006022].

[3] B. Lucini and M. Teper, Phys. Rev. D 64, 105019 (2001) [arXiv:hep-lat/0107007].

[4] B. Lucini and M. Teper, Phys. Rev. D 66, 097502 (2002) [arXiv:hep-lat/0206027].

[5] L. Del Debbio, H. Panagopoulos, P. Rossi and E. Vicari, JHEP 0201, 009 (2002) [arXiv:hep-th/0111090].

[6] L. Del Debbio, H. Panagopoulos and E. Vicari, JHEP 0309, 034 (2003) [arXiv:hep-lat/0308012].

[7] B. Lucini, M. Teper and U. Wenger, improved operators," JHEP 0406, 012 (2004) [arXiv:hep-lat/0404008].

[8] M. R. Douglas and S. H. Shenker, "Dynamics of SU(N) supersymmetric gauge theory," Nucl. Phys. B 447, 271 (1995) [arXiv:hep-th/9503163].

[9] A. Hanany, M. J. Strassler and A. Zaffaroni, Nucl. Phys. B 513, 87 (1998) [arXiv:hep-th/9707244].

[10] B. Svetitsky and L. G. Yaffe, Nucl. Phys. B 210, 423 (1982).

[11] D. Diakonov, N. Gromov, V. Petrov and S. Slizovskiy, Phys. Rev. D 70, 036003 (2004) [arXiv:hep-th/0404042].

[12] N. M. Davies, T. J. Hollowood, V. V. Khoze and M. P. Mattis, Nucl. Phys. B 559, 123 (1999) [arXiv:hep-th/9905015].

[13] N. M. Davies, T. J. Hollowood and V. V. Khoze, J. Math. Phys. 44, 3640 (2003) [arXiv:hep-th/0006011]. 